\title{
Dynamic range of cochlear implant stimulation is larger in residually hearing cochlea
}

\author{
Mika Sato* and Andrej Kral ${ }^{\dagger}$ \\ Institute of AudioNeuroTechnology \& Dept. of Experimental Otology, ENT Clinics, Hannover Medical University, \\ Stadtfelddamm 34, 30625 Hannover, Germany
}

(Received 30 September 2018, Accepted for publication 19 May 2019)

Keywords: Cochlear implant, Electrophonic response, Electroneural response PACS number: 43.64.Me [doi:10.1250/ast.41.380]

\section{Introduction}

Cochlear implants (CIs) were originally developed as prosthetic devices for patients with profound hearing loss. The criteria for their application have been progressively expanded to include patients with residual hearing at low frequencies. The combination of electrical stimulation at high frequencies and residual acoustical sensitivity at low frequencies improves speech understanding [1]. However, despite the clinical success, little is known about the physiological basis of this benefit.

Electric stimulation of a residually hearing cochlea generates a direct neural response (electroneural response, $\mathrm{EN}$ ) and a hair-cell-mediated response (electrophonic response, EP) [2]. The EN occurs at the position of the active electrode and the EP occurs at the dominant frequency component of the electrical stimulus time function [3]. The origin of the EP is unknown, but several possibilities have been discussed [4]. In this study, we focus on the dynamic range of these responses in an attempt to clarify the origin of the EP.

\section{Method}

Eleven adult guinea pigs were used for this study. All experimental procedures were approved by the local state authorities and were performed in compliance with the guidelines of the European Community for the care and use of laboratory animals (EU VD 86/609/EEC) and the German Animal Welfare Act (TierSchG).

The animals were kept under general anesthesia with intramuscular injection of $50 \mathrm{mg} / \mathrm{kg} 2 \%$ ketamin and xylazine and artificially ventilated through the tracheotomy using a rodent ventilator. The end-tidal $\mathrm{CO}_{2}$ concentration was kept below 4\%. The core temperature of the animals was monitored and kept above $38^{\circ} \mathrm{C}$ using a homeothermic blanket. The electrocardiogram, ventilation rate, end-tidal $\mathrm{CO}_{2}$, and core temperature were used to monitor the physiological status of the animals. Auditory brainstemevoked responses (ABRs) to condensation clicks $(50 \mu \mathrm{s})$ were used for hearing assessment.

A guinea pig CI (four contacts at intervals of $1 \mathrm{~mm}, \mathrm{MED}-$ EL $\mathrm{GmbH}$ ) was inserted into the cochlea through a cochleostomy. The electrical stimuli were single, charge-balanced,

\footnotetext{
*e-mail: Sato.Mika@mh-hannover.de

†e-mail: Kral.Andrej@mh-hannover.de
}

biphasic electric pulses $(100 \mu \mathrm{s} /$ phase $)$ with the current level controlled using attenuators.

A Neuronexus double-shank 32-channel electrode array was stereotactically placed within the inferior colliculus parallel to the tonotopic axis. Correct placement of the recording electrode array was confirmed by receptive-field measurement of unit activity (stimuli: tones, $500 \mathrm{~Hz}-32 \mathrm{kHz}$, four steps per octave, 0-90 dB sound pressure level (SPL), $5 \mathrm{~ms}$ on/off flanks, $50 \mathrm{~ms}$ duration, using a calibrated loudspeaker). The characteristic frequency ( $\mathrm{CF}$ ) of each recording site was determined and used for further analyses (initial mapping).

After the mapping of the $\mathrm{CF}$ of each recording site, a CI was inserted through a cochleostomy and the same mapping procedure was repeated to determine the change in hearing after implantation (postimplantation mapping). Responses to electric stimulation via the CI under the hearing condition were recorded before remeasuring the acoustic receptive fields to ensure the preservation of the hearing after electric stimulation. Thereafter, hair cells were destroyed by an intracochlear application of $100 \mu$ of $5 \%$ neomycin sulfate solution with the CI left untouched. After profound hearing loss was observed for the same stimuli as those used for the CF mapping, the same electrical stimulation was repeated under the deafened condition.

The data were analyzed offline. First, the matrix of the recorded signals was filtered for unit activity (high-pass, second-order elliptic filter; cutoff frequency $=400 \mathrm{~Hz}$ ). A threshold value for triggering action potentials was selected as exceeding 3 standard deviations above the mean noise level with a spike duration above $0.25 \mathrm{~ms}$.

To evaluate responsiveness, peristimulus time histograms with $1 \mathrm{~ms}$ bins obtained from 10 (acoustic) or 50 (electric) stimulus repetitions were compared between the hearing and deafened conditions, and the firing rate was expressed as the maximum instantaneous multiunit firing rate per millisecond within the response in the peristimulus time histogram.

Thresholds and rate-level functions were determined under each condition. The dynamic range was computed by subtracting the threshold current level from the level at which the response reached saturation. The two-tailed WilcoxonMann-Whitney test were used for significance testing.

\section{Result}

The hearing thresholds of all the animals were first 

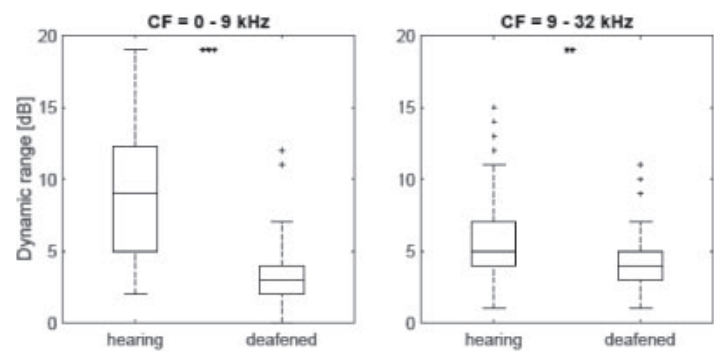

Fig. 1 Dynamic range under the hearing and deafened conditions for apical $(\mathrm{CF}<9 \mathrm{kHz})$ and basal $(\mathrm{CF} \geq 9$ $\mathrm{kHz}$ ) regions of the cochlea.

screened on the basis of their click-evoked ABRs. Only normally hearing animals (with hearing threshold $<30 \mathrm{~dB}$ SPL) were used in this study. Excellent hearing preservation after cochlear implantation was ensured by the post-CImapping (comp. [3]).

Electrical stimulation via the CI was performed using the most apical electrode and an indifferent (return) retroauricular electrode (monopolar configuration), and the responses in the inferior colliculus were recorded. The cochlea was subsequently pharmacologically deafened with the CI left in place and the same stimulation and recording were performed.

Data were pooled separately from positions with CFs below $9 \mathrm{kHz}$ (apical: beyond the reach of the CI electrode) and above $9 \mathrm{kHz}$ (basal: within the reach of the CI electrode). The dynamic range was computed and a statistical comparison was performed.

The mean dynamic ranges (Fig. 1) of the CFs below $9 \mathrm{kHz}$ were $8.9 \pm 4.3 \mathrm{~dB}$ under the hearing condition and $3.4 \pm 2.3 \mathrm{~dB}$ under the deafened condition. At the CFs above $9 \mathrm{kHz}$, the dynamic ranges were $5.8 \pm 3.0 \mathrm{~dB}$ and $4.6 \pm 2.1$ $\mathrm{dB}$, respectively. In both datasets, the dynamic range before deafening was significantly larger than that after deafening $(p<0.001$ at $\mathrm{CFs}$ below $9 \mathrm{kHz}, p<0.01$ at $\mathrm{CFs}$ above
$9 \mathrm{kHz}$; Wilcoxon-Mann-Whitney test). At CFs below $9 \mathrm{kHz}$, this difference was larger than that above $9 \mathrm{kHz}$.

\section{Conclusion}

In this study, we show that the dynamic range is larger when the cochlea contains hair cells (comp. also [5] for local field potentials). This allows better coding of the dynamic changes in sound pressure in the electrically stimulated ear. Additionally, the significant difference in the dynamic range between both of the frequency groups supports the hypothesis that the EP occurs in hearing cochlea regardless of the frequency region. As a mechanism, direct electrical stimulation of the outer hair cells is more likely than basilar membrane vibration or the depolarization of the inner hair cells. As a consequence, EP responses also occur beyond the best cochlear location, i.e., beyond the dominant frequency of the electrical stimulus time function [3].

\section{Acknowledgements}

Supported by Deutsche Forschungsgemeinschaft (Exc 1077).

\section{References}

[1] C. W. Turner, B. J. Ganz, A. Vidal Cehrens and B. A. Henry, "Speech recognition in noise for cochlear implant listeners: Benefits of residual acoustic hearing," J. Acoust. Soc. Am., 115, 1729-1735 (2004).

[2] E. C. Moxon, "Neural and mechanical responses to electric stimulation of the cat's inner ear," PhD Thesis, Massachusetts Institute of Technology (1971).

[3] M. Sato, P. Baumhoff and A. Kral, "Cochlear implant stimulation of a hearing ear generates separate electrophonic and electroneural responses," J. Neurosci., 36, 54-64 (2016).

[4] M. Sato, P. Baumhoff, J. Tillein and A. Kral, "Physiological mechanisms in combined electric-acoustic stimulation," Otol. Neurotol., 38, e215-e223 (2017).

[5] H. S. Lusted and F. B. Simmons, "Comparison of electrophonic and auditory-nerve electroneural responses," J. Acoust. Soc. Am., 83, 657-661 (1988). 